\title{
Experimental Research on Intratumoral Injection of Sal Ammoniac Extract in the Treatment of Mouse Liver Cancer
}

\author{
Yuanyuan Jia1, Chang Tian1, Siqi Wang1, Yu Feng1, Wujun Li², Yu Cai2* \\ ${ }^{1}$ Department of Teacher Development and Teaching Evaluation, The First Affiliated Hospital of Xi'an Medical University, \\ Xi'an 710000, Shaanxi Province, China \\ 2Department of General Surgery, The First Affiliated Hospital of Xi'an Medical University, Xi'an 710000, Shaanxi Province,
} China

*Corresponding author: Yu Cai, caiyu3721@ sina.com

\begin{abstract}
Objective: To study the effect of sal ammoniac extract on the treatment of liver cancer and analyze its possibility of replacing absolute ethanol. Methods: Sixty Kunming mice (5-6 weeks old, 18-22g in weight, male and female in half) were selected and inoculated with $0.1 \mathrm{ml}$ of 1:4 ascitic diluent from mouse liver cancer $\mathrm{H} 22$ under the axilla of the right limb. After tumor formation, they were randomly divided into 3 groups with 20 mice in each group. Normal saline (NS), sal ammoniac extract $(\mathrm{N})$, and absolute ethanol (E) were injected into the tumor once a day for four times. The death, tumor weight, tumor inhibition rate, lactate dehydrogenase (LDH), and succinate dehydrogenase (SDH) expression of the mice in each group were analyzed. Results: In the course of treatment, 3 mice died in the normal saline group, 2 mice died in the sal ammoniac treatment group, and 7 mice died in the absolute ethanol group. The tumor weight of the normal saline group, sal ammoniac treatment group, and absolute ethanol group were $1.88 \pm 0.26,1.41 \pm 0.49$, and $1.51 \pm 0.46$, respectively. The tumor inhibition rates were $0 \%, 31.0 \%$, and $21.6 \%$, respectively. Comparing the sal ammoniac treatment group and the normal saline group, $\mathrm{t}$ $=3.5133, p=0.0013$; comparing the absolute ethanol group and the normal saline group, $\mathrm{t}=2.7926, p=0.0093$. The expression of LDH was $81 \pm 10,51 \pm 11$, and $32 \pm 9$ in the normal saline group, sal ammoniac treatment group, and absolute ethanol group, respectively, while the expression of SDH was $80 \pm 10,51 \pm 10$, and $51 \pm 12$ in the normal saline group, sal ammoniac treatment group, and absolute ethanol group, respectively. Comparing the LDH of the sal ammoniac treatment group with that of the saline group, $\mathrm{t}=8.4264, p=0.0000$; comparing the absolute ethanol group and normal saline group, $\mathrm{t}$ $=13.8763, p=0.0000$. Comparing the SDH of the sal ammoniac treatment group with that of the normal saline group, $\mathrm{t}=$ $8.1455, p=0.0000$; comparing the absolute ethanol group with the normal saline group, $\mathrm{t}=7.2197, p=0.0000$. Conclusion: The traditional Chinese antitumor medicine, sal ammoniac and its main effective components have good antitumor effect, which can be further popularized and applied in clinical practice.
\end{abstract}

Keywords: Sal ammoniac extract; Traditional Chinese medicine; Tumor; Liver cancer

Publication date: September 2021; Online publication: September 30, 2021

\section{Introduction}

Liver cancer is a common malignant tumor. China is one of the many countries with high incidence of liver cancer around the world. The comprehensive treatment of liver cancer is an important research topic in clinical practice. In regard to that, the local treatment of liver cancer has become an important development trend ${ }^{[1,2]}$. 
Sal ammoniac has the functions of eliminating accumulation and removing blood stasis, softening hardness, and dispersing knot. It can be used for the treatment of swelling of welling-abscesses, which is equivalent to the treatment of cutaneous furuncle with abscess, folliculitis, acute suppurative lymphadenitis, acute suppurative cellulitis, suppurative arthritis, and bone tuberculosis in modern medicine. Because it has the function of resolving hard lump, it can be used for the treatment of ocular pterygium, nasal polyps, esophageal malignant tumors, gastric malignant tumors, and abdominal tumors ${ }^{[3]}$.

In this article, the effect of a traditional Chinese medicine, sal ammoniac extract, on the treatment of liver cancer is analyzed along with determining the possibility of the extract replacing absolute ethanol.

\section{Materials and methods}

\subsection{Experimental materials}

Sixty Kunming mice (5-6 weeks old, 18-22g in weight, male and female in half) provided by Heilongjiang Cancer Research Institute were used in this study (certificate number: 050621). Hepatoma H22 was also provided by Heilongjiang Cancer Research Institute. The drugs and reagents used in this study were the compound preparation of sal ammoniac extract (composed of sal ammoniac, Chinese Stellera root, Radix glycorrhizae, etc.), provided by Xinchan Research Institute of Traditional Chinese Medicine, containing 12 $\mathrm{g} / \mathrm{ml}$ crude medicine and stored in the refrigerator at $4^{\circ} \mathrm{C}$.

\subsection{Experimental methods}

$0.1 \mathrm{ml}$ of 1:4 ascitic diluent from mouse liver cancer $\mathrm{H} 22$ was inoculated under the axilla of the right limb of the mice. Tumor formation took 4 days. Thereafter, they were randomly divided into 3 groups with 20 mice in each group. Normal saline (NS), sal ammoniac extract (N), and absolute ethanol (E) were injected into the tumor once a day, four times in total. After 4 days, the mice were killed, the tumor tissues were stripped, weighed, and the tumor inhibition rate was calculated. Tumor inhibition rate $=$ (tumor weight of control group - tumor weight of experimental group) / tumor weight of control group. Samples were taken for histochemical staining for lactate dehydrogenase (LDH) and succinate dehydrogenase (SDH).

\subsection{Statistical methods}

Statistical Package for the Social Sciences (SPSS) version 22.0 was used to analyze and process the relevant data. The counting data was expressed in percentage (\%), the comparison was expressed by chi-square $\left(\chi^{2}\right)$ test, and the measurement data was expressed by t-test. The difference was considered statistically significant when $p<0.05$.

\section{Results}

\subsection{Death of mice in each group}

In the course of treatment, 3 mice died in the normal saline group, 2 mice died in the sal ammoniac treatment group, and 7 mice died in the absolute ethanol group.

\subsection{Tumor inhibition in each group}

When the mice were examined after a period of treatment, the results showed that the tumor weight of the normal saline group, sal ammoniac treatment group, and absolute ethanol group were $1.88 \pm 0.26,1.41 \pm$ 0.49 , and $1.51 \pm 0.46$, respectively. The tumor inhibition rates were $0 \%, 31.0 \%$, and $21.6 \%$, respectively. See Table 1 for details. Comparing sal ammoniac treatment group with the normal saline group, $\mathrm{t}=3.5133$, $p=0.0013$; comparing absolute ethanol group with the normal saline group, $\mathrm{t}=2.7926, p=0.0093$. 
Table 1. Tumor inhibition of the mice in each group

\begin{tabular}{lcc}
\hline \multicolumn{1}{c}{ Group } & Tumor weight $(\bar{x} \pm s)$ & Tumor inhibition rate $(\%)$ \\
\hline Normal saline group $(\mathrm{n}=17)$ & $1.88 \pm 0.26$ & 0 \\
Sal ammoniac treatment group $(\mathrm{n}=18)$ & $1.41 \pm 0.49$ & 31.0 \\
Absolute ethanol group $(\mathrm{n}=13)$ & $1.51 \pm 0.46$ & 21.6 \\
\hline
\end{tabular}

\subsection{Expression of LDH and SDH in each group}

When the mice were examined after a period of treatment, the results showed that the expression of LDH was $81 \pm 10,51 \pm 11$, and $32 \pm 9$ in the normal saline group, sal ammoniac treatment group, and absolute ethanol group, respectively, while the expression of SDH was $80 \pm 10,51 \pm 10$, and $51 \pm 12$, respectively. See Table 2 for details. Comparing the LDH in the sal ammoniac treatment group with the saline group, $\mathrm{t}$ $=8.4264, p=0.0000$; comparing the absolute ethanol group with the normal saline group, $\mathrm{t}=13.8763, p=$ 0.0000. Comparing the SDH in the sal ammoniac treatment group with the normal saline group, $\mathrm{t}=8.1455$, $p=0.0000$; comparing the absolute ethanol group with the normal saline group, $\mathrm{t}=7.2197, p=0.0000$.

Table 2. Expression of LDH and SDH in the mice in each group

\begin{tabular}{lcc}
\hline \multicolumn{1}{c}{ Group } & LDH $(\bar{x} \pm s)$ & SDH $(\bar{x} \pm s)$ \\
\hline Normal saline group $(\mathrm{n}=17)$ & $81 \pm 10$ & $80 \pm 10$ \\
Sal ammoniac treatment group $(\mathrm{n}=18)$ & $51 \pm 11$ & $51 \pm 10$ \\
Absolute ethanol group $(\mathrm{n}=13)$ & $32 \pm 9$ & $51 \pm 12$ \\
\hline
\end{tabular}

\section{Discussion}

According to the latest global tumor statistics released by the World Health Organization, the incidence of liver cancer cases in China accounts for $45.3 \%$ and the number of deaths accounts for $47.1 \%$ of the world. About $80 \%$ of patients with liver cancer are already at the middle or advanced stage once they have been diagnosed, indicating that they do not have the option for radical resection and their 5-year survival rate is less than $18 \%$. Therefore, the implementation of a more accurate and effective targeted therapy has always been the focus and difficulty in the clinical and basic research of liver cancer ${ }^{[4-6]}$.

Various local treatments for HCC were performed via percutaneous transhepatic procedure. It is a major progress in the field of interventional therapy for HCC in recent years. In the early 1990s, several scholars applied this treatment method to the treatment of large residual HCC lesions via hepatic artery embolization and achieved excellent results. Its mechanism involves tumor tissue necrosis by the high concentration of ethanol causing cell dehydration, protein coagulation, and tumor vascular occlusion ${ }^{[7,9]}$. However, due to the limited infiltration capacity of ethanol, more absolute ethanol, multiple punctures, and multiple courses of treatment are needed to ensure a tumor is completely necrotic. This inevitably increases the side effects and complications, as well as the possibility of needle tract implantation. In order to improve the efficacy and reduce the times of injection, sal ammoniac extract was used in this study as a local injection in the treatment of the transplanted tumor in vivo. It has achieved good tumor suppressive effect in mice. The results showed that the tumor inhibition rate of sal ammoniac extract in mice was higher than that of absolute ethanol. During the treatment, the survival rate of the mice in the sal ammoniac group was higher than that in the absolute ethanol group, suggesting that the toxicity of sal ammoniac extract is lower than that of anhydrous ethanol. Histochemical staining showed that sal ammoniac extract was similar to absolute ethanol, which reduces the expression of lactate dehydrogenase (LDH) and succinate dehydrogenase (SDH), as two important metabolic enzymes in the development of liver cancer, and directly 
inhibit the growth of liver cancer.

In conclusion, the traditional Chinese antitumor medicine, sal ammoniac, and its main effective components have good antitumor effect, which can be further popularized and applied in clinical practice.

\section{Funding}

This research was supported by the General and Special Scientific Research Project of the Education Department of Shaanxi Province (Project Number: 20JK0887): Molecular Mechanism of MiR-122 Competitive Endogenous LncRNA in the Development of Liver Cancer.

\section{Disclosure statement}

The authors declare that there is no conflict of interest.

\section{References}

[1] Liang Y, Shen T, 2021, The Role and Mechanism of Mir-22 and Its Target Genes in Hepatocellular Carcinoma. Chemistry of Life, 41(06): 1252-1258.

[2] Zhuo ET, Zheng YC, Fu GH, et al., 2021, Clinical Effect of Hepatic Arterial Chemoembolization Combined with Absolute Ethanol Ablation in Elderly Patients with Primary Liver Cancer. Chinese Journal of Gerontology, 41(14): 2956-2958.

[3] Deng SR, Liu NJ, Li FY, et al., 1997, Qualitative Certification on the Traditional Chinese Medicine Sal Ammoniac. China Journal of Chinese Materia Medica, 22(05): 259-261.

[4] Luo XQ, Ding GM, Zheng X, et al., 2021, Improvement of the Animal Model of Orthotopic Transplantation of Hepatic Carcinoma in Mice. Chinese Journal of Comparative Medicine, 31(06): 1622.

[5] Liu XH, Liu FY, 2021, Bioinformatics Analysis and Construction of autophagy Gene related Hepatocellular Carcinoma prognosis Assessment Model. Zhejiang Medicine, 43(12): 1320-1324, 1328, 1371.

[6] Wang X, 2021, Comparative Study of RECIST1.1, mRECIST and qEASL Tumor Evaluation Criteria in the Evaluation of the Efficacy of TACE Combined with Microwave Ablation for Primary Liver Cancer. Northwest Minzu University.

[7] Li WD, Guo XD, Chen JL, et al., 2021, Ct-Guided Percutaneous Absolute Ethanol Injection Combined with Radiofrequency Ablation for Primary Liver Cancer in High-Risk Sites. Chinese Journal of Minimally Invasive Surgery, 21(07): 605-609.

[8] Wang H, 2021, Study on the Function and Mechanism of Long Chain Noncoding RNA GIMA Promoting the Survival of Liver Cancer Cells under Glucose Starvation. University of Science and Technology of China.

[9] Tang HX, Li SS, Hong F, et al., 2021, Construction of a Novel Human Hepatocellular Carcinoma Xenotransplantation Model in Normal Immunized Mice. Journal of Clinical Hepatol, 37(11): 1-5. 